\title{
A Patient with Escherichia coli Bacteremia and COVID-19 Co-Infection: A Case Report for the Louisville COVID-19 Epidemiology Study
}

\author{
Sarah E. Moore ${ }^{1 *}$, PharmD; Ashley M. Wilde ${ }^{1}$, PharmD BCPS-AQ ID; Matthew Song ${ }^{1}$, PharmD BCIDP; Brian C. Bohn ${ }^{1}$, PharmD; Clayton J. \\ Patross $^{1}$, PharmD BCPS; Bryan Denham ${ }^{2}, M D ;$ Paul Schul ${ }^{3}, M D$; Julio A. Ramire ${ }^{4}, M D$ \\ ${ }^{1}$ Norton Pharmacy Services, Norton Healthcare, Louisville, KY, USA, ${ }^{2}$ Norton Audubon Hospital, Norton Healthcare, Louisville, KY, USA, ${ }^{3}$ Norton Infectious Diseases \\ Specialists, Norton Healthcare, Louisville, KY, USA , ${ }^{4}$ Division of Infectious Diseases, Department of Medicine, School of Medicine, University of Louisville, Louisville, \\ KY, USA \\ *sarah.moore@nortonhealthcare.org
}

Recommended Citation: Moore SE, Wilde AM, Song M, et al. A patient with Escherichia coli bacteremia and COVID-19 co-infection: A case report for the Louisville COVID-19 Epidemiology Study. Univ Louisville J Respir Infect 2020; 4(1):Article 15. doi: 10.18297/jri/vol4/iss1/15.

\begin{abstract}
Patients with COVID-19 may have co-infections with other microorganisms. Here we report a case of a patient with an Escheria coli bacteremia secondary to a urinary tract infection, who experienced fevers while on active antimicrobial therapy.

\section{Introduction}

Current CDC guidance encourages health care providers to consider testing for SARS-CoV-2 infection in patients with signs and symptoms consistent with COVID infection.[1] Reports of both viral and bacterial co-infection are now emerging.[2-6] We describe a patient with Escherichia coli bacteremia secondary to a urinary tract infection, who developed persistent high fevers despite receiving appropriate antimicrobial therapy based on susceptibility results. Due to clinical deterioration, the patient was tested for novel coronavirus infection and found to have a positive nasopharyngeal PCR. Our case report serves as a caution against ruling out SARS-CoV-2 when another infectious process may not fully explain a patient's symptoms.
\end{abstract}

The patient was eventually tested for COVID-19 and found to be positive. This case emphasizes the need to suspect COVID-19 even in patients with documented bacterial infection.

\section{Case Presentation}

A 64-year-old white male presented to the emergency department with a chief complaint of burning with urination starting 4 days prior to admission. His past medical history was significant for type 2 diabetes mellitus, hypertension and pancreatitis. The patient also endorsed urinary retention, gait instability, nausea, vomiting and chills. Blood and urine cultures were collected, both of which grew pan-susceptible E. coli. The patient received active antibiotics for his $E$. coli infec- tion throughout his hospitalization.

On hospital day 2, the patient had a urinary catheter placed due to continued urinary retention. He also reported diarrhea and was tested for $C$. difficile infection, which was negative. Repeat blood cultures were obtained and demonstrated no growth, confirming clearance of the patient's bacteremia.

On hospital day 4, a CT of the abdomen/pelvis was obtained, which noted an evolving prostate abscess (Figure 1). Of note, the patient also began reporting a dry cough and developed high fevers with a maximum temperature of $103.4^{\circ} \mathrm{F}$. That evening, he required $\mathrm{Bi}$ PAP in order to maintain $\mathrm{SaO}_{2}>90 \%$. He was eventually intubated due to respiratory failure. At this point, a nasopharyngeal COVID-19 PCR test was obtained due to rapid clinical deterioration despite adequate management of the patient's bacterial infection. The patient was also placed into COVID-19 appropriate isolation.

The patient went on to develop multi-organ failure, including acute respiratory distress syndrome (Figure 2 ), and required continuous renal replacement therapy and multiple vasopressors to maintain MAP $>65$ mmHg. On hospital day 9, the COVID-19 PCR test resulted positive, confirming COVID-19 coinfection. At this time, the patient was non-responsive off sedation and was compassionately extubated shortly thereafter. 


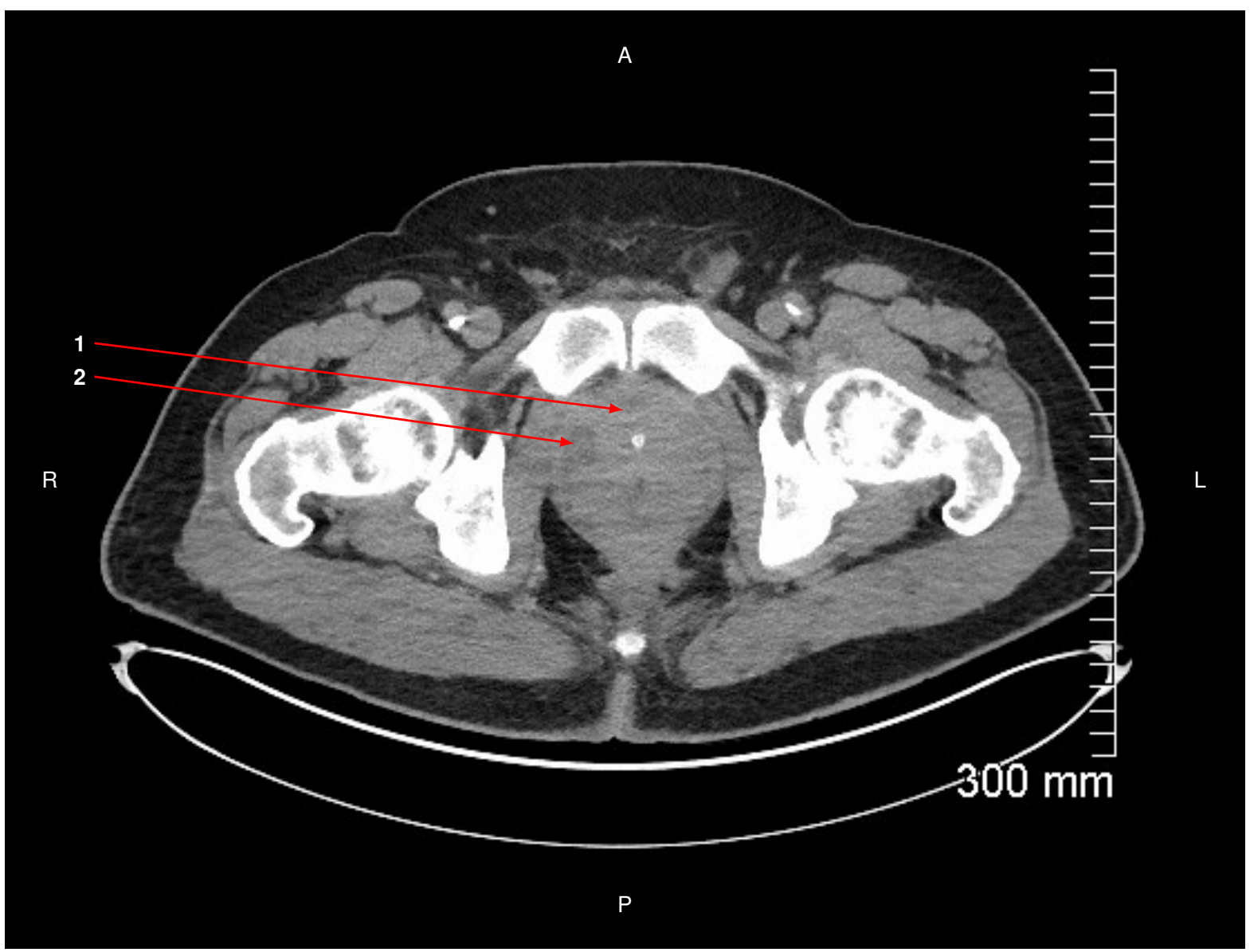

Figure 1. CT abdomen/pelvis obtained on hospital day 2. Findings significant for 1) enlarged prostate, seminal vesicles and thick-walled urinary bladder (not visualized here), and 2) new focal $2 \times 1.3 \mathrm{~cm}$ region of decreased attenuation at the right lateral periphery of the prostate. It is unclear if this represents phlegmon/early abscess in the setting of prostatitis.

\section{Discussion}

SARS-CoV-2 can present with a constellation of nonspecific symptoms. In our patient, nausea and diarrhea were possibly the first COVID-19 related symptoms to present. This was followed by a non-productive cough, fevers, and eventual respiratory decompensation.[7, 8] Initially, the patient's fevers while on antibiotics were thought to be attributable to his undrained prostate abscess. However, given that this patient was initially afebrile and then developed high fevers while receiving antibiotics, this raised suspicion for a second infectious process. The median incubation period of SARSCoV-2 appears to be about 5 days.[9-11] Lauer et al. reported that in their cohort, $<2.5 \%$ of patients displayed any symptoms within 2.2 days.[9] Based on this information, it seems most likely that our patient acquired COVID-19 prior to presenting to the hospital.

Guidelines from the Surviving Sepsis Campaign suggest that based on current evidence, the presence of another respiratory pathogen does not necessarily rule out the possibility of COVID-19.[12] In this case, there was delay in clinical suspicion and COVID-19 testing. During the first four days of hospitalization, the patient was not in isolation resulting in numerous healthcare worker exposures. This highlights the importance of the aforementioned recommendation. Universal patient screening upon admission may have identified this patient as a COVID-19 case earlier.

Clinical outcomes in patients with COVID-19 and bacterial coinfections have not been well-described. Fan and colleagues describe a case of a patient with COVID-19 and Mycoplasma pneumoniae infection.[3] These authors suggest that failing to treat other causes of infection in COVID-19 patients is likely to increase morbidity and mortality.

Worse outcomes were observed in patients with bacterial coinfection and H1N1 influenza. These patients were more likely to require mechanical ventilation and longer ICU stays than patients without coinfection.[13] Additionally, patients with bacterial coinfection had an 


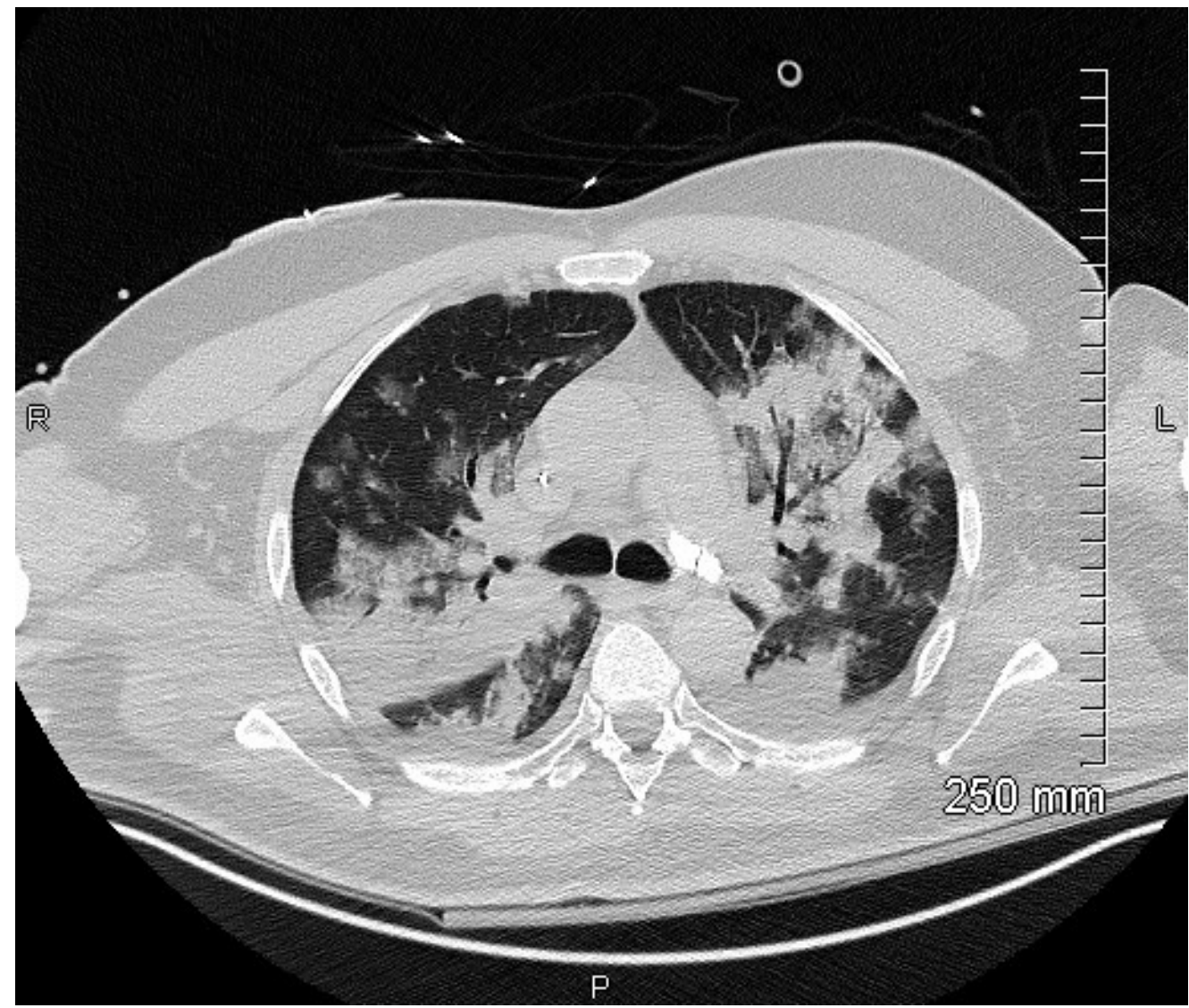

Figure 2. CT chest obtained on hospital day 5. Findings include extensive bilateral dense consolidation involving the upper and lower lobes. There is a small left pleural effusion. Overall appearance is consistent with widespread pneumonia and acute respiratory distress syndrome (ARDS).

inpatient mortality rate of $31 \%$, versus $21 \%$ in patients with influenza alone $(P=0.002)$. [13] It is possible that similar outcomes may be observed in the SARS-CoV-2 pandemic, but further data is needed before any conclusions may be drawn.
In conclusion, this case highlights the importance of considering COVID-19 infection even in the setting of a confirmed alternate infectious diagnosis. Clinicians should consider isolation and testing for coinfection if clinically appropriate.
Received: April 27, 2020

Accepted: May 20, 2020

Published: June 4, 2020

Copyright: () 2022 The author(s). This original article is brought to you for free and open access by ThinkIR: The University of Louisville's Institutional Repository. For more information, please contact thinkir@louisville.edu. This article is distributed under the terms of the Creative Commons Attribution 4.0 International License (CC BY 4.0), which permits unrestricted use, distribution, and reproduction in any medium, provided the original author and source are credited.

Funding Source: The author(s) received no specific funding for this work.

Conflict of Interest: All authors declared no conflict of interest in relation to the main objective of this work. 


\section{References}

1. Centers for Disease Control and Prevention. Evaluating and testing persons for coronavirus disease 2019 (COVID19). Available at: https://www.cdc.gov/coronavirus/2019nCoV/hcp/clinical-criteria.html. Accessed 20 April 2020.

2. Touzard-Romo F, Tapé C, Lonks JR. Co-infection with SARS-CoV-2 and human metapneumovirus. R I Med J (2013) 2020; 103(2):75-6. PMID: 32192233.

3. Fan BE, Lim KGE, Chong VCL, Chan SSW, Ong $\mathrm{KH}$, Kuperan P. COVID-19 and Mycoplasma pneumoniae coinfection. Am J Hematol 2020; 95(6):723-4. doi: 10.1002/ajh.25785. PMID: 32173883.

4. Khodamoradi Z, Moghadami M, Lotfi M. Co-infection of coronavirus disease 2019 and influenza A: A report from Iran. Arch Iran Med 2020; 23(4):239-43. doi: 10.34172/aim.2020.04. PMID: 32271596.

5. Xing Q, Li G-j, Xing Y-h, et al. Precautions are needed for COVID-19 patients with coinfection of common respiratory pathogens. SSRN [Preprint]. 2020 doi: 10.2139/ssrn.3550013.

6. Kim D, Quinn J, Pinsky B, Shah NH, Brown I. Rates of co-infection between SARS-CoV-2 and other respiratory pathogens. JAMA 2020; 323(20):2085-6. doi: 10.1001/jama.2020.6266. PMID: 32293646.

7. Sun $\mathrm{Y}, \mathrm{Koh} \mathrm{V}$, Marimuthu $\mathrm{K}$, et al. Epidemiological and clinical predictors of COVID-19. Clin Infect Dis 2020; 71(15):78692. doi: 10.1093/cid/ciaa322. PMID: 32211755.
8. D'Amico F, Baumgart DC, Danese S, Peyrin-Biroulet L. Diarrhea during COVID-19 infection: Pathogenesis, epidemiology, prevention, and management. Clin Gastroenterol Hepatol 2020; 18(8):1663-72. doi: 10.1016/j.cgh.2020.04.001. PMID: 32278065.

9. Lauer SA, Grantz $\mathrm{KH}, \mathrm{Bi} \mathrm{Q}$, et al. The incubation period of coronavirus disease 2019 (COVID-19) from publicly reported confirmed cases: Estimation and application. Ann Intern Med 2020; 172(9):577-82. doi: 10.7326/m20-0504. PMID: 32150748.

10. Linton NM, Kobayashi T, Yang $Y$, et al. Incubation period and other epidemiological characteristics of 2019 novel coronavirus infections with right truncation: A statistical analysis of publicly available case data. J Clin Med 2020; 9(2). doi: 10.3390/jcm9020538. PMID: 32079150.

11. Li Q, Guan X, Wu P, et al. Early transmission dynamics in Wuhan, China, of novel coronavirus-infected pneumonia. N Engl J Med 2020; 382(13):1199-207. doi: 10.1056/NEJMoa2001316. PMID: 31995857.

12. Alhazzani $\mathrm{W}$, Møller MH, Arabi YM, et al. Surviving sepsis campaign: Guidelines on the management of critically ill adults with coronavirus disease 2019 (COVID-19). Intensive Care Med 2020; 46(5):854-87. doi: 10.1007/s00134-02006022-5. PMID: 32222812.

13. Rice TW, Rubinson L, Uyeki TM, et al. Critical illness from 2009 pandemic influenza A virus and bacterial coinfection in the United States. Crit Care Med 2012; 40(5):1487-98. doi: 10.1097/CCM.0b013e3182416f23. PMID: 22511131. 